\title{
The moderating effects of mindfulness facets on psychological distress and emotional eating behaviour
}

\section{BACKGROUND}

Emotional eating is an impulsive mood-regulation strategy that often follows psychological distress. Mindfulness is associated with less impulsive behaviour. Mindful eating involves a considered awareness of hunger and satiety, and conscious, non-automatic, food choices. This study examines the moderating role of mindfulness on the relationship between distress and emotional eating.

\section{PARTICIPANTS AND PROCEDURE}

Participants $(N=392)$ completed self-report measures on distress, mindfulness and emotional eating, after which moderation analysis was carried out.

\section{RESULTS}

Mindfulness was negatively associated with emotional eating, but only when distress was low. The most important facets of mindfulness for this were being able to describe one's emotional state and a non-judgemental response to that state.

\section{CONCLUSIONS}

These results support previous findings that mindfulness reduces the impact distress has on emotional eating. Future research could explore interventions that enable individuals to describe their emotional state in the moment to reduce preoccupation with food during times of distress.

\section{KEY WORDS}

mindfulness; emotional eating; stress; anxiety; depression

organization - Department of Psychology, Sociology and Politics, Sheffield Hallam University, Sheffield,

United Kingdom

AUthors' CONTRibutions - A: Study design - B: Data collection - C: Statistical analysis - D: Data interpretation .

E: Manuscript preparation · F: Literature search · G: Funds collection

Corresponding author - Diarmuid Verrier, Ph.D., Department of Psychology, Sociology and Politics, Sheffield Hallam University, Howard Str., Sheffield S1 1WB, United Kingdom, e-mail: d.verrier@shu.ac.uk

TO CITE THIS ARTICLE - Verrier, D., \& Day, C. (2022). The moderating effects of mindfulness facets on psychological

distress and emotional eating behaviour. Health Psychology Report, 10(2), 103-110. https://doi.org/10.5114/

hpr.2021.109921

RECEIVED 26.05.2021 · REVIEWED 30.07.2021 · ACCEPTED 08.09.2021 · PUBLISHED 18.10.2021 


\section{BACKGROUND}

Making modifications to diet and lifestyle is an obvious solution to reduce the health problems associated with overeating and making poor food choices. However, due to the complexities of eating behaviour, reducing intake and making healthier food choices are often easier said than done. For example, the onset of stress or negative affect can result in changes to our eating habits, such as overeating or undereating (see for example Wallis \& Hetherington, 2009). This is a manifestation of emotional eating (van Strien et al., 1986) and it can make it particularly challenging for individuals to make positive changes to their diet.

Those high in emotional eating are more prone to worry about eating, engage in higher food monitoring, and experience less perceived control over their eating and food choices compared to those low in emotional eating (see review by Bongers \& Jansen, 2016). When experiencing stress and anxiety, being more mindful, or 'in the moment', may reduce worry about eating and those feelings of loss of control of eating. Mindfulness is a state of non-judgmental awareness of one's current experience, more formally defined as "paying attention in a particular way, on purpose, in the present moment, and nonjudgmentally" (Kabat-Zinn, 1994, p. 4). Mindfulness is associated with less impulsive behaviour which Shapiro and Carlson (2009) describe as a combination of mindful awareness and mindful practice. Research on mindfulness has significantly increased over the last several decades, and it has been found that higher trait mindfulness and mindfulness practice has many positive outcomes for health and well-being (e.g., Mantzios \& Giannou, 2019; Sala et al., 2020). One of the areas in which mindfulness has been shown to be beneficial is eating behaviour (e.g., Tapper, 2017). Mindful eating stems from general theories of mindfulness; as such it might follow that mindful eating involves 'being in the moment' when selecting and consuming food. However, as outlined in a recent review (see Mantzios, 2020), there are many challenges in reaching a formal, widely agreed definition of mindful eating.

A number of studies have shown that interventions and training designed to increase mindfulness can usefully reduce dysfunctional eating behaviours such as binge eating (see review by Grohmann \& Laws, 2021, for examples). Apart from interventions and training, however, high dispositional or trait mindfulness has also been shown to be associated with less emotional eating (Ouwens et al., 2015). One aspect of emotional eating is a maladaptive coping mechanism wherein people eat in order to reduce negative affect. Essentially, emotional cues such as anger, anxiety, and depression evoke eating responses in addition to typical physiological hunger cues. Given that mindfulness entails a non-judgemental awareness of one's internal emotional, cognitive, and physiological state in the present moment, it could potentially serve as a self-regulatory tool that allows individuals to avoid automatic emotion-related eating responses. For example, in those with low levels of mindfulness, emotional distress may positively correlate with emotional eating, while high mindfulness could serve to disrupt the automatic eating response that follows negative affect, so that distress and emotional eating do not correlate (i.e., a moderating relationship between distress and mindfulness).

The first study to explicitly test this possible moderation effect was carried out by Pidgeon et al. (2013). They recruited 157 participants (a mixture of student and community participants) who completed measures of distress (Depression, Anxiety, and Stress Scales; Lovibond \& Lovibond, 1995), trait mindfulness (Mindfulness Awareness Attention Scale; Brown \& Ryan, 2003), and emotional eating (a subscale of the Three Factor Eating Questionnaire; Karlsson et al., 2000). They found that there was an interaction effect between mindfulness and distress on emotional eating, though it differed from the one sketched out above. Specifically, high mindfulness was associated with less emotional eating only when distress levels were low - at higher levels of distress the protective effects of mindfulness dissipated. Looking at the components of distress separately, the authors found that this same interaction was found for depression alone, but not anxiety or stress. This is a valuable finding, but it comes with two caveats. First, the study may have been slightly underpowered to have full confidence in the finding (Button et al., 2013). A post hoc power analysis using reported figures from the study suggests that the power to detect the interaction effect was only about.4. Second, they used a unidimensional measure of mindfulness that conceptualises mindfulness as present-centred attention-awareness. A more recent measure that was developed via factor analysis of the items from a number of pre-existing mindfulness instruments has suggested that mindfulness may be better conceptualised as having five distinct facets, defined as nonreactivity, observing, acting with awareness, nonjudging and describing (Baer et al., 2006).

Three studies have been published that did use a multidimensional mindfulness scale (Five Facet Mindfulness Questionnaire; Baer et al., 2006) and were thus able to obtain more fine-grained detail as to which aspects of mindfulness were relevant to emotional eating. Tak et al. (2015) found the expected relationship between mindfulness and emotional eating in a sample of 634 adults with diabetes: higher levels of mindfulness were associated with lower emotional eating. Closer examination of the facets revealed that 'acting with awareness' was the strongest individual predictor of lower levels of emotional and external eating, followed by 'describing' and 'being 
non-judgemental'. Lattimore et al. (2011) found the same three facets of mindfulness to significantly correlate with emotional eating in a sample of 190 women (student and community sample). More recently, Höppener and colleagues (2019) explored whether depressive symptoms and emotional eating were mediated by mindfulness in a large adult sample. They found that of the five mindfulness facets, only 'acting with awareness' mediated the association between depressive symptoms and emotional eating. While these findings confirm that there is an association between dispositional mindfulness, emotional eating and depression, none of these studies has investigated whether mindfulness moderated the association between negative affect and emotional eating despite gathering data on anxiety and depression.

In keeping with current meta science trends relating to replication, the current study explores the moderating effect of mindfulness on the association between emotional distress and emotional eating, following Pidgeon et al.'s (2013) study. However, we extend this to employ a larger sample to gain adequate statistical power. We also use a multidimensional measure of mindfulness in order to determine which specific facets of mindfulness are responsible for any observed moderation effect. In line with Pidgeon et al. (2013), it is hypothesised that there will be an interaction effect between overall mindfulness and emotional distress on emotional eating. More speculatively, it is hypothesised that the three mindfulness facets (acting with awareness, describing, and being non-judgemental) identified by Tak et al. (2015) and Lattimore et al. (2011) will also be shown to moderate distress.

\section{PARTICIPANTS AND PROCEDURE}

\section{PARTICIPANTS}

The study was completed by a convenience sample of 392 participants from the UK (115 men, 276 women, and 1 who self-identified as transsexual). The age range was from 17 to 72 years $(M=24.20, S D=10.10)$. The mean body mass index (BMI) of the sample was within the healthy weight range $\left(M=23.40 \mathrm{~kg} / \mathrm{m}^{2}\right.$, $S D=4.18$, range $16-38 \mathrm{~kg} / \mathrm{m}^{2}$ ). The sample was a mixture of participants who came from the general community and students. The study was disseminated through social media channels, snowballing and the university student participant pool. Some students received course credit for participation.

\section{MEASURES}

Depression, Anxiety, and Stress Scales. Distress was assessed using the 21-item version of the Depression,
Anxiety, and Stress Scales (DASS; Lovibond \& Lovibond, 1995). Items are positively worded and use a self-report 4-point response scale. For each item participants must rate the extent to which they have experienced a particular state or feeling over the last week. Internal reliability is reported by the authors to be .91 for the depression scale and .84 for the anxiety scale. The stress scale, which relates to feelings of nervous tension, irritability, and agitation, has a reported internal reliability of .90 . We found similarly good internal reliability (Cronbach's $\alpha$ of $.90, .84$, and .85 respectively).

Five Facet Mindfulness Questionnaire. Mindfulness was measured using the Five Facet Mindfulness Questionnaire (FFMQ; Baer et al., 2006). Items use a self-report 5-point Likert scale; some are negatively worded. This scale has five factors: awareness (e.g., "I find it difficult to stay focused on what's happening in the present"), observing (e.g., "When I'm walking I deliberately notice the sensation of my body moving"), non-judgement (e.g., "I criticize myself for having irrational or inappropriate emotions"), non-reactivity (e.g., "I perceive my feelings and emotions without having to react to them"), and describing (e.g., "I'm good at finding words to describe my feelings"). A systematic review by Park et al. (2013) reported that the internal reliability for these scales ranges from .67 to .93 . We also found the overall scale to have good internal consistency (Cronbach's $\alpha=.85$ ).

Three Factor Eating Questionnaire. Emotional eating was assessed using the relevant subscale of the Three Factor Eating Questionnaire (TFEQ-EE; Karlsson et al., 2000). This has three, positively worded, items, which use a 4-point Likert response scale (e.g., "When I feel anxious, I find myself overeating"). In line with the authors, we found the Cronbach's $\alpha$ for this scale to be .85 .

\section{PROCEDURE}

Ethical approval for this study was granted by the university's research ethics committee. Thereafter, participants were recruited through advertisements, word of mouth, and internal departmental research participation recruitment processes. The questionnaire was hosted online. The actual scales were preceded by an information sheet outlining the purpose of the study and drawing attention to key ethical principles, which participants read before deciding to progress with the study.

\section{ANALYSIS}

Zero-order correlations were performed to explore the linear relationships between the main variables
Mindfulness, psychological distress and emotional eating 
Catherine Day, Diarmuid Verrier prior to moderation analysis. Moderation analysis was carried out using the PROCESS macro within SPSS (see www.processmacro.org). These analyses evaluated the interaction effect between distress and mindfulness on emotional eating. Assuming an effect size of comparable magnitude to that observed by Pidgeon et al. (2013; i.e., $f^{2}=.04$ ), a study wishing to have power of .8 would need a sample size of approximately 365 to reliably detect an interaction between mindfulness and distress (calculations performed using $G^{*}$ Power; Faul et al., 2009). The sample size used here (392) exceeds that.

\section{RESULTS}

The data were assessed for normality and showed a small amount of positive skew on the DASS scores. However, given the relatively large sample size, skewness was not considered problematic and data were deemed suitable for parametric analysis (ClarkCarter, 2009).

Descriptive statistics and bivariate correlations (Pearson's $r$ ) between the variables can be seen in Table 1. As expected, all of the DASS subscales correlate well with each other. The FFMQ subscales correlate less with each other. Most strikingly, observing and non-judgement correlate negatively with each other (a similar relationship was found by the original authors; Baer et al., 2006). However, all of the subscales correlate at least moderately with the overall FFMQ score. As expected, overall DASS correlates positively with emotional eating, as do two of its subscales (anxiety and depression). Overall FFMQ correlates negatively with emotional eating, as do all of its subscales (other than observing).

Following this, moderation analysis was carried out using the PROCESS macro within SPSS. These analyses assessed the interaction effect between distress and mindfulness on emotional eating. The first analysis assessed the interaction between overall distress and mindfulness and included DASS-total, FFMQ-total, and the interaction term for these two variables (i.e., their product after the variables were centred). The overall model was found to be significant, $R^{2}=.07, F(3,385)=9.88, p<.001$. Mindfulness was a significant predictor of emotional eating $(b=-.37,95 \%$ CI $[-.61,-.14], p=.002)$ while distress was not. These effects were dependent upon a significant interaction term, $b=.50,95 \%$ CI $[.14, .86]$, $p=.007$, which was responsible for an increase in $R^{2}$ of .02. Figure 1 shows a simple slope representation of this interaction, wherein lower distress is related to less emotional eating, but only in those with higher mindfulness.

Having shown that overall mindfulness and distress interact, the second set of analyses looked at the interaction between overall mindfulness and each of the distress components separately. The same pattern was found for depression, anxiety, and stress as was found for overall distress. That is, they did not independently predict emotional eating, but the interactions between depression and mindfulness $(b=.35$, $95 \%$ CI $[.06, .65], p=.020)$, and anxiety and mindfulness $(b=.35,95 \%$ CI $[.06, .64], p=.020)$, were both significant. In each case, lower negative affect was

Table 1

Descriptive statistics and correlations for the main variables

\begin{tabular}{|c|c|c|c|c|c|c|c|c|c|c|c|}
\hline & \multirow[t]{2}{*}{$M(S D)$} & \multicolumn{10}{|c|}{ Correlations } \\
\hline & & 2 & 3 & 4 & 5 & 6 & 7 & 8 & 9 & 10 & 11 \\
\hline 1. DASS-total & $1.91(0.60)$ & $.90^{* *}$ & $.86^{* *}$ & $.88^{* *}$ & $-.49^{* *}$ & $.14^{* *}$ & $-.30^{* *}$ & $-.45^{* *}$ & $-.57^{* *}$ & $-.12^{*}$ & $.13^{*}$ \\
\hline 2. DASS-D & $1.80(0.72)$ & & $.65^{* *}$ & $.70^{* *}$ & $-.52^{* *}$ & .07 & $-.36^{* *}$ & $-.45^{* *}$ & $-.54^{* *}$ & $-.11^{*}$ & $.11^{*}$ \\
\hline 3. DASS-A & $2.16(0.68)$ & & & $.61^{* *}$ & $-.41^{* *}$ & $.13^{* *}$ & $-.20^{* *}$ & $-.39^{* *}$ & $-.51^{* *}$ & $-.13^{*}$ & $.14^{* *}$ \\
\hline 4. DASS-S & $1.76(0.66)$ & & & & $-.36^{* *}$ & $.18^{* *}$ & $-.22^{* *}$ & $-.35^{* *}$ & $-.47^{* *}$ & -.09 & .08 \\
\hline 5. FFMQ-total & $3.12(0.43)$ & & & & & $.39^{* *}$ & $.72^{* *}$ & $.65^{* *}$ & $.52^{* *}$ & $.48^{* *}$ & $-.23^{* *}$ \\
\hline 6. FFMQ-O & $3.13(0.75)$ & & & & & & $.26^{* *}$ & -.08 & $-.29^{* *}$ & $.28^{* *}$ & .02 \\
\hline 7. FFMQ-D & $3.33(0.79)$ & & & & & & & $.36^{* *}$ & $.14^{* *}$ & $.21^{* *}$ & $-.11^{*}$ \\
\hline 8. FFMQ-A & $3.11(0.79)$ & & & & & & & & $.45^{* *}$ & -.02 & $-.19^{* *}$ \\
\hline 9. FFMQ-NJ & $3.08(0.86)$ & & & & & & & & & .01 & $-.20^{* *}$ \\
\hline 10. FFMQ-NR & $2.94(0.67)$ & & & & & & & & & & $-.16^{* *}$ \\
\hline 11. TFEQ-EE & $2.29(0.87)$ & & & & & & & & & & \\
\hline
\end{tabular}


associated with less emotional eating but only in those with higher mindfulness. The interaction between stress and mindfulness also showed this pattern but fell just short of the adopted significance level $(b=.35,95 \%$ CI $[.00, .71], p=.050)$.

The next step was to evaluate the moderating role of each of the mindfulness facets separately. As the general pattern of moderation did not greatly depend on which distress component was used as a moderator, overall distress was used to predict emotional eating alongside each mindfulness facet. For the observing facet, there was no main effect on emotional eating, nor was there an interaction with distress. The awareness facet showed a main effect on emotional eating, $b=-.17,95 \%$ CI $[-.29$, $-.04], p=.008$, but no interaction with distress. The same was true of the non-reacting facet, which also showed a main effect on emotional eating, $b=-.18$, $95 \%$ CI $[-.31,-.05], p=.008$, but no significant interaction with distress, although it was close to significance $(p=.054)$. In those cases where there was a main effect, higher mindfulness was associated with less emotional eating.

The remaining facets, non-judgement and describing, were both found to interact with distress (these significant interactions are fully reported in Table 2). For the interactions between distress and the describing facet, and between distress and the non-judgement facet, the pattern was the same as that observed for distress and total mindfulness (see Figure 1). That is, describing and non-judgement were associated with less emotional eating, but only in those with low overall distress. The sizes of the increases in $R^{2}$ represented by these interactions were small $\left(f^{2}=.02\right.$; Cohen, 1988).

\section{DISCUSSION}

The present study considered whether dispositional mindfulness moderates the association between distress and emotional eating in a large non-clinical sample. We found that there was a moderating effect wherein mindfulness was negatively associated with emotional eating, but only when distress was low; at high distress, there was no difference in emotional eating between those with high and low mindfulness. Put another way, emotional eating was low when distress was low and mindfulness was high. Thus, it seems as though having a mindful awareness of oneself, whether in relation to one's emotional state or

\section{Figure 1}

Interaction effect between distress and mindfulness on emotional eating

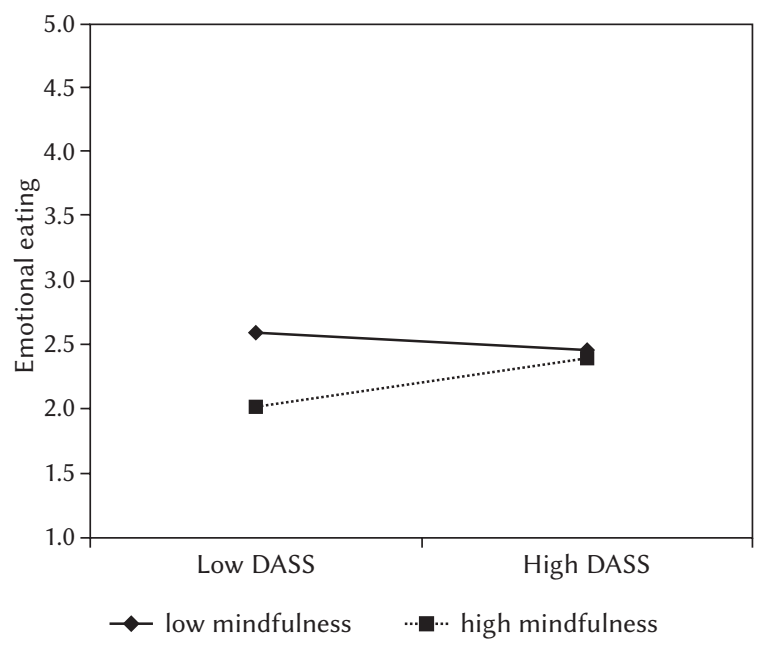

Mindfulness, psychological distress and emotional eating

\section{Table 2}

Descriptive statistics for the sample

\begin{tabular}{|c|c|c|c|c|c|c|c|c|}
\hline & \multicolumn{4}{|c|}{ FFMQ - Describing (moderator) } & \multicolumn{4}{|c|}{ FFMQ - Non-judgement (moderator) } \\
\hline & $b[95 \% \mathrm{Cl}]$ & $S E$ & $t$ & $p$ & $b[95 \% \mathrm{Cl}]$ & $S E$ & $t$ & $p$ \\
\hline Constant & $\begin{array}{c}2.32 \\
{[2.24,2.41]}\end{array}$ & .05 & 51.23 & $<.001$ & $\begin{array}{c}2.35 \\
{[2.25,2.44]}\end{array}$ & .05 & 48.52 & $<.001$ \\
\hline Distress (centred) & $\begin{array}{c}.17 \\
{[.02, .32]}\end{array}$ & .08 & 2.26 & .020 & $\begin{array}{c}.11 \\
{[-.07, .29]}\end{array}$ & .09 & 1.16 & .247 \\
\hline $\begin{array}{l}\text { FFMQ moderator } \\
\text { (centred) }\end{array}$ & $\begin{array}{c}-.09 \\
{[-.21, .02]}\end{array}$ & .06 & -1.57 & .120 & $\begin{array}{c}-.16 \\
{[-.28,-.04]}\end{array}$ & .06 & -2.60 & .010 \\
\hline \multirow[t]{2}{*}{$\begin{array}{l}\text { Interaction (Distress } \\
\times \text { FFMQ moderator) }\end{array}$} & $\begin{array}{c}.24 \\
{[.06, .43]}\end{array}$ & .09 & 2.61 & .009 & $\begin{array}{c}.20 \\
{[.05, .35]}\end{array}$ & .08 & 2.65 & .008 \\
\hline & \multicolumn{4}{|c|}{$R^{2}=.04$} & \multicolumn{4}{|c|}{$R^{2}=.06$} \\
\hline
\end{tabular}

Note. Only the regression analyses with significant interactions are reported here. The dependent variable is emotional eating, the independent variable is distress (DASS mean) and the moderators are the lower facets of the Five Facet Mindfulness Questionnaire (FFMQ). 
Catherine Day, Diarmuid Verrier one's response to eating/satiety cues, can effectively protect against depression and anxiety-related emotional eating, but only when those emotional states are relatively mild.

Unlike previous work in this area (Pidgeon et al., 2013), which found that only depression was moderated, we found that this moderating effect applied to both depression and anxiety. This is also the first study on moderation to use a multifactorial measure of mindfulness, which enabled us to discern which aspects of mindfulness are most relevant to this interaction effect. Tak et al. (2015) and Lattimore et al. (2011) previously found main effects for acting with awareness, describing, and non-judgement on emotional eating, while we found that non-reactivity was also able to predict emotional eating. Non-reactivity is accomplished through allowing experiences to come and go without reacting in an effort to change them. In this context, people who are more non-reactive will be aware of their negative emotional state but will be able to accept it as transient. As such, they will not feel the need to engage in emotional eating behaviour to assuage their negative emotions. Our findings may differ from previous studies due to the different samples used; Tak et al. (2015) focused on adults with diabetes, whilst Lattimore et al.'s (2011) sample was all-female. Our study reports data on a large community sample, including males and females, potentially making it more applicable to the general population.

Höppener et al. (2019) explored the association between mindfulness, depressive symptoms and emotional eating using a multifactorial measure, but their focus was on the mediating effect of this relationship. They found that the only facet to mediate the association between depressive symptoms and emotional eating was acting with awareness. Following these previous studies, we hypothesised that acting with awareness, describing, and non-judgement might also moderate the effect of psychological distress on emotional eating. Two of these three mindfulness facets were found to interact with distress: describing and non-judgement, but not acting with awareness, moderated the effect of distress on emotional eating. In both cases, the pattern was the same as that observed for overall mindfulness.

The use of the FFMQ, a multifactorial measure of mindfulness, is one of the strengths of this study. Although most frequently measured as a one-dimensional construct (Tomlinson et al., 2018), there is an increasing awareness that dispositional mindfulness is best assessed using multifaceted measures (Rau \& Williams, 2016). However, whether it makes sense to use a measure of dispositional mindfulness to assess mindfulness in an eating context is a different matter. Several authors have attempted to create scales that specifically measure mindful eating, with various degrees of success (see Mantzios, 2020, for a brief review). A fundamental problem in this endeavour is definitional complexity. For example, mindful eating is often construed as allowing people to make better decisions about eating, but this involves an evaluation of satiety and hunger cues, whereas a mindful perspective is intrinsically nonevaluative. To resolve this issue, Mantzios (2020) suggests separating mindful eating behaviour (attention to the taste and texture of food, alongside a non-judgemental awareness of thoughts related to eating that food) from the decisions that take place prior to eating. Future work on mindful eating needs to attend to these issues carefully so that there is no ambiguity in what is being measured.

The fact that this study replicates the main findings of Pidgeon et al. (2013) is important given the current focus on replication in the field of psychology. The effect sizes of the interactions here were smaller than those in the original study, but this is often the case in replications (see Schäfer \& Schwarz, 2019). Future studies should also seek to further replicate and expand upon these findings in other contexts and with other populations. The participants in this study were largely young and white, and fewer than a third of participants were men. This limits the degree to which we can be confident that this observed association between mindfulness and emotional eating is a universal phenomenon. Exploring mindfulness in clinical populations would also be useful as previous research has found that participants with bulimia and binge/purge type anorexia had substantially higher emotional eating scores than healthy controls and that emotional eating was a powerful predictor of binge eating (Ricca et al., 2012). As such, evaluating the possible moderating role of mindfulness in these contexts could be of enormous value. For research with clinical populations, it would probably be appropriate to use a different measure of emotional eating than that used here. The TFEQ-EE relates solely to emotional overeating, whereas emotional eating is likely to manifest quite differently in a sample of, say, people with anorexia (Ricca et al., 2012). Further, all three items on the scale refer to negative emotions, whereas more recent conceptualisations of emotional eating also encompass positive emotions (see review, Bongers \& Jansen, 2016).

Future studies might also seek to take a longitudinal approach, which would permit evaluation of the effect of dispositional mindfulness over time, or to use experimental methods to better consider causality. This might entail allocating participants to different emotion manipulation conditions or different mindfulness enhancing conditions (though cultivated mindfulness is thought to be quite distinct from dispositional mindfulness; Rau \& Williams, 2016).

Overall, the current study supports the notion that higher levels of mindfulness can help reduce depression and anxiety-related emotional eating in a large, 
non-clinical, community sample. As well as the value inherent in replicating previously observed effects, this study made further valuable contributions to the field by identifying a theoretically sensible role for the non-reactivity component of mindfulness in predicting emotional eating, and for clarifying the specific facets of mindfulness that are responsible for moderating the relationship between distress and emotional eating: describing and non-judgement. These two facets of mindfulness in particular are likely to be associated with a reflective, dispassionate cognitive style that buffers against negative affect and enhances emotional processing and regulation (Tomlinson et al., 2018), and should be a particular focus for future work in this area.

\section{References}

Baer, R. A., Smith, G. T., Hopkins, J., Krietemeyer, J., \& Toney, L. (2006). Using self-report assessment methods to explore facets of mindfulness. Assessment, 13, 27-45. https://doi.org/10.1177/ 1073191105283504

Bongers, P., \& Jansen, A. (2016). Emotional eating is not what you think it is and emotional eating scales do not measure what you think they measure. Frontiers in Psychology, 7, 1932. https://doi. org/10.3389/fpsyg.2016.01932

Brown, K. W., \& Ryan, R. M. (2003). The benefits of being present: Mindfulness and its role in psychological well-being. Journal of Personality and Social Psychology, 84, 822-848. https://doi.org/ 10.1037/0022-3514.84.4.822

Button, K. S., Ioannidis, J. P. A., Mokrysz, C., Nosek, B. A., Flint, J., Robinson, E. S. J., \& Munado, M. R. (2013). Power failure: Why small sample size undermines the reliability of neuroscience. Nature Reviews: Neuroscience, 14, 365-376. https:// doi.org/10.1038/nrn3475

Clark-Carter, D. (2009). Quantitative psychological research: The complete student's companion. Taylor and Francis.

Cohen, J. E. (1988). Statistical power analysis for the behavioral sciences. Lawrence Erlbaum Associates, Inc.

Faul, F., Erdfelder, E., Buchner, A., \& Lang, A. G. (2009). Statistical power analyses using $G^{*}$ Power 3.1: Tests for correlation and regression analyses. Behavior Research Methods, 41, 1149-1160. https:// doi.org/10.3758/BRM.41.4.1149

Grohmann, D., \& Laws, K. R. (2021). Two decades of mindfulness-based interventions for binge eating: a systematic review and meta-analysis. Journal of Psychosomatic Research, 149, 110592. https://doi. org/10.1016/j.jpsychores.2021.110592

Höppener, M. M., Larsen, J. K., van Strien, T., Ouwens, M. A., Winkens, L. H., \& Eisinga, R. (2019). Depressive symptoms and emotional eating: Me- diated by mindfulness? Mindfulness, 10, 670-678. https://doi.org/10.1007/s12671-018-1002-4

Kabat-Zinn, J. (1994). Wherever you go, there you are: Mindfulness meditation in everyday life. Hyperion Books.

Karlsson, J., Persson, L. O., Sjöström, L., \& Sullivan, M. (2000). Psychometric properties and factor structure of the Three-Factor Eating Questionnaire (TFEQ) in obese men and women. Results from the Swedish Obese Subjects (SOS) study. International Journal of Obesity, 24, 1715-1725. https://doi.org/10.1038/sj.ijo.0801442

Lattimore, P., Fisher, N., \& Malinowski, P. (2011). A cross-sectional investigation of trait disinhibition and its association with mindfulness and impulsivity. Appetite, 56, 241-248. https://doi.org/ 10.1016/j.appet.2010.12.007

Lovibond, P. F., \& Lovibond, S. H. (1995). The structure of negative emotional states: Comparison of the Depression Anxiety Stress Scales (DASS) with the Beck Depression and Anxiety Inventories. Behaviour Research and Therapy, 33, 335-343. https:// doi.org/10.1016/0005-7967(94)00075-U

Mantzios, M. (2020). (Re)defining mindful eating into mindful eating behaviour to advance scientific enquiry. Nutrition and Health. https://doi. org/10.1177/0260106020984091

Mantzios, M., \& Giannou, K. (2019). A real-world application of short mindfulness-based practices: a review and reflection of the literature and a practical proposition for an effortless mindful lifestyle. American Journal of Lifestyle Medicine, 13, 520-525. https://doi.org/10.1177/1559827618772036

Ouwens, M. A., Schiffer, A. A., Visser, L. I., Raeijmaekers, N. J., \& Nyklíček, I. (2015). Mindfulness and eating behaviour styles in morbidly obese males and females. Appetite, 87, 62-67. https://doi.org/ 10.1016/j.appet.2014.11.030

Park, T., Reilly-Spong, M., \& Gross, C. R. (2013). Mindfulness: a systematic review of instruments to measure an emergent patient-reported outcome (PRO). Quality of Life Research, 22, 2639-2659. https://doi.org/10.1007/s11136-013-0395-8

Pidgeon, A., Lacota, K., \& Champion, J. (2013). The moderating effects of mindfulness on psychological distress and emotional eating behaviour. Australian Psychologist, 48, 262-269. https://doi. org/10.1111/j.1742-9544.2012.00091.x

Ricca, V., Castellini, G., Fioravanti, G., Lo Sauro, C., Rotella, F., Ravaldi, C., Lazzeretti, L., \& Faravelli, C. (2012). Emotional eating in anorexia nervosa and bulimia nervosa. Comprehensive Psychiatry, 53, 245-251. https://doi.org/10.1016/j.comppsych. 2011.04.062

Rau, H. K., \& Williams, P. G. (2016). Dispositional mindfulness: a critical review of construct validation research. Personality and Individual Differences, 93, 32-43. https://doi.org/10.1016/j.paid.2015.09.035
Mindfulness, psychological distress and emotional eating 
Sala, M., Rochefort, C., Lui, P. P., \& Baldwin, A. S. (2020). Trait mindfulness and health behaviours: a meta-analysis. Health Psychology Review, 14, 345393. https://doi.org/10.1080/17437199.2019.1650290

Schäfer, T., \& Schwarz, M. A. (2019). The meaningfulness of effect sizes in psychological research: Differences between sub-disciplines and the impact of potential biases. Frontiers in Psychology, 10, 813. https://doi.org/10.3389/fpsyg.2019.00813

Shapiro, S. L., \& Carlson, L. E. (2009). The art and science of mindfulness: Integrating mindfulness into psychology and the helping professions. APA Publications.

Catherine Day, Diarmuid Verrier

Tak, S. R., Hendrieckx, C., Nefs, G., Nyklíček, I., Speight, J., \& Pouwer, F. (2015). The association between types of eating behaviour and dispositional mindfulness in adults with diabetes. Results from Diabetes MILES. The Netherlands. Appetite, 87, 288-295. https://doi.org/10.1016/j.appet.2015.01.006

Tapper, K. (2017). Can mindfulness influence weight management related eating behaviors? If so, how? Clinical Psychology Review, 53, 122-134. https:// doi.org/10.1016/j.cpr.2017.03.003

Tomlinson, E., Yousaf, R., Vittersø, O., \& Jones, A. (2018). Dispositional mindfulness and psychological health: a systematic review. Mindfulness, 9, 2343. https://doi.org/10.1007/s12671-017-0762-6

van Strien, T., Frijters, J. E., Bergers, G. P., \& Defares, P. B. (1986). The Dutch Eating Behavior Questionnaire (DEBQ) for assessment of restrained, emotional, and external eating behavior. International Journal of Eating Disorders, 5, 295-315. https://doi.org/10.1002/1098-108X(198602)5:2<295: :AIDEAT2260050209>3.0.CO;2-T

Wallis, D. J., \& Hetherington, M. M. (2009). Emotions and eating. Self-reported and experimentally induced changes in food intake under stress. Appetite, 52, 355-362. https://doi.org/10.1016/j.appet. 2008.11.007 